\title{
Interacciones de dos tipos de habitantes del campo al Norte del Valle de Colima (México)*
}

\author{
Fecha de recepción: 15 de mayo de 2013 Fecha de aceptación: 24 de noviembre de 2013 Disponible en línea: 30 de julio de 2014 \\ Victor Valladares Vadillo \\ \begin{tabular}{l|l|l} 
Magister en Arquitectura & Estudiante de la Universidad de Colima & arqvictorvalladares@hotmail.com
\end{tabular}
}

Francisco Javier Cárdenas Munguía

\begin{tabular}{|l|l|}
\hline Doctor en Arquitectura & Profesor e investigador de gestión urbana en el ordenamiento territorial \\
\hline
\end{tabular}

Universidad de Colima fjcardenasm@hotmail.com

Resumen El artículo aborda un tema de impacto por los fenómenos de la urbanización dispersa en el territorio rural y toma como caso de estudio la zona norte del Valle de Colima (México). A su vez, busca caracterizar los impactos sociales en los habitantes originarios de la zona en comparación con los modos de vida de los nuevos habitantes de costumbres urbanas. Se hace un análisis teórico que incluye conceptos como el desarrollo rural, la urbanización del campo, la calidad de vida, el desarrollo endógeno y la ambitectura, entre otros; luego se particulariza la zona de estudio y se describen los fenómenos de la urbanización y, por último, se indaga la percepción de los habitantes originarios y urbanos sobre la calidad de vida en la región.

Palabras clave Desarrollo rural; interacción campo-ciudad; percepción social; suelo rural-campestre; territorio rural; urbanización del campo

\footnotetext{
Artículo de investigación científica y tecnológica titulada "Transformaciones socioterritoriales en comunidades campestres del norte del municipio de Villa de Álvarez, Colima". Fue desarrollado en el programa de Maestría en Arquitectura con área terminal en desarrollo urbano en la Universidad de Colima, financiado por el Consejo Nacional de Ciencia y Tecnología (Conacyt); inició en agosto de 2011 y culminó en julio de 2013. La línea de investigación es gestión urbana en el ordenamiento territorial, del cuerpo académico consolidado UCOL-CA 27: Arquitectura y ciudad.
}

Cómo citar este artículo: Valladares-Vadillo, V. y Cárdenas-Munguía, F. J. (2014). Interacciones de dos tipos de habitantes del campo al norte del Valle de Colima, (México). Cuadernos de Vivienda y Urbanismo, 7(14), 242-259. http://dx.doi.org/10.11144/Javeriana.CVU7-14.idth 


\section{Interactions between Two Rural Residents at North of the Valley of Colima (Mexico)}

Abstract The article approach a topic of impact phenomen of urban sprawl in the rural territory and takes as a case study the northern Valley Colima, Mexico. In turn, seeks to characterize the social impacts on the original inhabitants of the area compared to the lifestyles of the new inhabitants of urban customs. A theoretical analysis that includes concepts such as rural development, rural urbanization, urbanization of the country, endogenous development, ambitectura, inter alia, then the study area is characterized and the phenomena of development are described, and finally have the perception that original and urban dwellers in relation to the quality of life in the region is investigated.

Keywords Rural-country land; rural development; rural territory; rural-urban interaction; social perception; urbanization of the country

\section{Interação de dois tipos de pessoas no campo North Valley Colima (México)}

Resumo $\mathrm{O}$ artigo discute um tema de impacto phenomen da expansão urbana no território rural e tem como estudo de caso no norte do Vale Colima, no México. Por sua vez, procura caracterizar os impactos sociais sobre os habitantes originais da área, em comparação com o estilo de vida dos novos moradores de costumes urbanos. Uma análise teórica que inclui conceitos como desenvolvimento rural, a urbanizaçáo rural, qualidade de vida, o desenvolvimento endógeno, ambitectura, e outros, em seguida, a área de estudo é caracterizada e os fenômenos de desenvolvimento são descritos, e, finalmente, ter a percepção de que original e urbana moradores em relação à qualidade de vida na região é investigada.

Palavras chave Desenvolvimento rural; interação rural-urbana; percepção social;

terra rural-piquenique; território rural; urbanização do campo 


\section{Introducción}

Este artículo muestra una visión acerca del encuentro entre la ciudad y el medio rural y las consecuencias sociales que lo rodean. En el continente americano, en especial en países de rápido crecimiento demográfico como los nuestros, este tema refleja una problemática cotidiana de nuestras urbanizaciones. Familias acomodadas y de estratos sociales medios y medios altos invaden sectores rurales empobrecidos con condominios y estructuras habitacionales cerradas que poco o nada comparten con las comunidades locales, lo que genera bolsones inertes de dormitorio y fines de semana que impactan en las formas de vidas y costumbres rurales patrimoniales. La vida de campo desaparece para dar paso a un dormitorio de campo.

Se ofrece un análisis de información teórica y conceptual para abordar el fenómeno de estudio de la ocupación de edificaciones campestres ${ }^{1}$ en comunidades rurales, así como las interacciones socioterritoriales que se establecen entre los habitantes de las comunidades campestres del norte del municipio de Villa de Álvarez (NMVA), en el estado de Colima (México).

Indagar en la investigación de fenómenos que tienen presencia en zonas rurales en las que se manifiestan relaciones sociales específicas es una tarea compleja, debido a que los estudios rurales, a diferencia de los urbanos, requieren ser planteados desde enfoques y metodologías específicas. En este sentido, es necesario tener presentes los saberes empíricos, teóricos y conceptuales sobre el territorio rural, así como las actividades, los modos de vida ${ }^{2}$ y su importancia.

Como problemática de estudio se detectaron las relaciones sociales establecidas entre los habitantes originarios de las comunidades y los habitantes atraídos a este territorio particular; de igual manera, se identificó que en los últimos veinte años han aparecido fraccionamientos campestres ${ }^{3}$ en la zona de estudio y, por lo tanto, una ocupación espacial que ha provocado diversos cambios en las comunidades donde se localizan.

Las transformaciones en el territorio y en las relaciones sociales ocasionadas por este fenómeno de inserción urbana en el suelo rural conforman la temática particular que se cubre en este trabajo. Se hizo un análisis de caso en la región norte del valle de Colima, para comprender la aparente existencia de una polarización de la economía, que se revela en el tipo de viviendas que ocupan el espacio y definen el territorio rural, ahora modificado.

Se inicia con el planteamiento hipotético: los procesos de desarrollo rural de las comunidades en el territorio norte del municipio de Villa de Álvarez del estado de Colima están siendo

1 Viviendas de segunda residencia, casas de campo, casas de descanso.

2 Se propone la evaluación de las condiciones de vida en una zona rural como el eje principal de estudio; para ello, se revisó el desarrollo comunitario del territorio rural, debido a que los vértices económicos, ambientales y sociales se ven involucrados en las transformaciones del territorio, ocasionadas por la inserción urbana en el suelo rural.

3 Se consideran zonas habitacional-campestres las que están fuera de los límites de los centros de población, a una distancia no menor de tres kilómetros de ellos, de acuerdo con el Reglamento de zonificación del estado de Colima. 
afectados por un fenómeno socioterritorial resultante de la manifestación de la urbanización del campo. En esa zona coexisten dinámicas urbanas y rurales que generan una dicotomía en la lectura del ambiente en los aspectos territoriales, espaciales y sociales.

Para comprobar la hipótesis, se diseñó una metodología que plantea la construcción de un perfil sobre las condiciones de vida de los habitantes originarios y campestres, como alternativa para la medición del desarrollo comunitario del territorio rural.

Evaluar la percepción de las condiciones de vida mediante la lectura de los activadores sociales, culturales y económicos del territorio englobados en la percepción de los tipos de habitantes de las comunidades - en cuanto a sus índices de satisfacción del bienestar- nos permite entender las diferencias en las perspectivas de cada uno e identificar los segmentos sociales que se ven directamente afectados por las condiciones de relación. Asimismo, el estudio de las variaciones de la zona de estudio desde la lectura del ambiente territorial, espacial y social en el análisis sistémico de la organización campo-ciudad facilita conocer los impactos generados en los contextos rurales característicos de la región.

En la revisión de la literatura no se detectó alguna metodología concreta que abordara mediciones del desarrollo comunitario rural, por lo que su desarrollo es una de las principales aportaciones de este trabajo.

A continuación listamos los pasos seguidos en el desarrollo de la investigación. En cada uno de ellos intervienen otros ítems necesarios para la elaboración del estudio:

1. Definición del espacio para la investigación (lectura del ambiente espacial)

- Universo de estudio: territorio rural del norte del municipio de Villa de Álvarez.
- Unidades de análisis: seis asentamientos humanos en contexto rural del norte del municipio de Villa de Álvarez, vinculados por el eje vial que define la carretera que conecta el centro de Villa de Álvarez con la comunidad de El Naranjal. Corresponde a El Chivato, Las Joyitas, El Carrizal, Nuevo Naranjal, La Lima y El Naranjal.

- Lectura de los patrones espaciales de las edificaciones de habitantes originarios y de las edificaciones de habitantes campestres.

2. Caracterización del territorio de las comunidades de estudio (lectura del ambiente territorial)

- Datos de la población (densidad/total de habitantes censados/Crecimiento porcentual 2000-2010).

- Forma de agrupación de la comunidad (dispersa/agrupada).

- Fraccionamientos campestres en el contexto de las comunidades.

- Comunidades con edificaciones campestres dentro del asentamiento originario.

3. Caracterización de las interacciones socioterritoriales en las comunidades (análisis de la ecoforma)

- Total de edificaciones originarias y campestres.

- Clasificación de la localización de edificaciones campestres dentro del territorio.

- Determinación de una muestra estadística de acuerdo con los porcentajes de edificaciones originarias y edificaciones campestres.

4. Diseño y aplicación de cuestionarios sobre la percepción de las condiciones de vida (lectura de la socioforma)

- Levantamiento de información en cada unidad de análisis. 
- Aplicación de cuestionarios sobre la percepción de las condiciones de vida de los habitantes.

5. Obtención de valores, sistematización y análisis de resultados (interpretación de la morfogénesis)

6. Elaboración de conclusiones

\section{Revisión conceptual y teórica}

Se considera como primer punto a revisar la definición del territorio de ocupación de las ciudades como un espacio finito que requiere una delimitación política y administrativa. Esto implica el esclarecimiento de una zona de transición en donde se establece una frontera que divide el campo de la ciudad; es una línea imaginaria que solo existe en los planes urbanos oficiales.

Lo que queda fuera de esta línea se localiza en la periferia urbana. En esta zona, se expresan fenómenos sociales y espaciales que se repiten de manera continua e interminable sobre el contorno de cualquier urbe, independientemente de su aptitud para el desarrollo urbano o del deterioro ambiental que se pueda ocasionar (Bazant, 2010, p. 476). Es una dinámica línea virtual, que dificulta establecer límites entre lo rural y lo urbano.

Más que con el crecimiento demográfico de las ciudades, el fenómeno de ocupación del suelo periférico a las ciudades se revela con la absorción de territorio rural para usos urbanos que experimentan las ciudades. De aquí que se conceptualice este límite no como una línea, sino como una franja de transición en la que hay viviendas dispersas entre áreas de cultivo, es decir, con ambos usos del suelo: urbano y rural. Es un proceso permanente de expansión y consolidación urbana, en el cual el territorio rural es ocupado.

En este contexto, se entendería que la planeación urbana debe incorporar las dimensiones políticas, culturales y ambientales, en el supuesto de que las ciudades constituyen, además de complejos sistemas productivos y ecológicos, la forma cultural más avanzada en que la sociedad se organiza y se reproduce (Olivera, 2004, p. 4); no son solo áreas o puntos en el territorio. El conjunto de fenómenos que se presentan en el territorio corresponden a una visión particular de las características que se manifiestan en cada asentamiento humano y en su contexto geográfico.

Delgado (2003) considera la existencia de dos escalas geográficas al analizar el crecimiento o la expansión de la ciudad sobre el ámbito rural inmediato: la producida por la expansión de la periferia conurbada y la generada por la expansión fragmentada no conurbada. Mientras la primera corresponde a la incorporación territorial de las zonas periféricas inmediatas al asentamiento humano mayor, la segunda agrega fragmentos territoriales a la zona urbana inmersos entre el contexto territorial rural. De ahí se distingue la presencia de algunos procesos económicos, sociales o demográficos particulares.

De la interacción entre lo urbano y lo rural se deriva una serie de conflictos que ocurre en todos los ámbitos, incluidas la familia, la comunidad y la región; tiene lugar, sobre todo, en las disputas por usos distintos del suelo: residencial y agrícola (Drescher e Iaquinta, 2000, p. 26). Esta paulatina profusión de actividades no agrícolas en zonas rurales, distantes de los núcleos urbanos, lleva a una valoración distinta del campo y requiere un enfoque distinto para su análisis (Hiernaux, 2001, p. 32).

De esta forma se establece una relación entre los asentamientos humanos de comunidades rurales con la cultura y los procesos propios que históricamente ocuparon los contextos agrícolas, al ser alcanzados por la expansión fragmentada no conurbada de las zonas urbanas. En este contexto, la ruralidad adquiere una nueva dimensión de estudio y una revaloración en cuanto a la dinámica 
de contacto entre la población ligada a las actividades agropecuarias y la desarrollada en funciones de ciudad.

La incursión de viviendas de tipo campestre en contextos rurales periféricos de las ciudades es resultado de una implantación en suelo que recién se hace urbano, ya sea a partir de fraccionamientos campestres o por la edificación campestre dentro del territorio de asentamientos rurales, debido al interés de las personas en la valoración del paisaje rural y del estilo de vida campestre.

Esta situación genera conflictos por los cambios en los usos de suelo del territorio que pueden darse a partir de la obtención del dominio pleno de la parcela ejidal, donde el propietario puede enajenar las tierras a terceros para usos urbanos ${ }^{4}$ (Cruz, 2002, p. 39). Como proceso previo, la obtención del dominio pleno puede derivar en la enajenación de parcelas. El suelo rural que haya obtenido el dominio pleno de la propiedad obtiene valor comercial, porque puede ser "desarrollado" para usos campestres, de manera que es una opción para "urbanizar el campo" por el uso de suelo campestre.

De igual forma, los cambios en el uso del suelo han repercutido en la asignación de una mayor responsabilidad a las instituciones que regulan los procesos de crecimiento urbano, lo que puede llevar a una relación más conflictiva entre procesos urbanos y rurales (Cruz, 1996, p. 142). Para diagnosticar esta relación se requiere obtener información con el fin de valorar los distintos impactos y las modificaciones en el contexto rural de las áreas de reserva, así como evidenciar la falta de planeación urbana integral que controle y regule el crecimiento, con un orden en la incorporación de nuevos desarrollos en los sitios de transición entre lo urbano y lo rural.

En este contexto de la gestión y del control del desarrollo urbano por parte del Estado y de apropiación por parte de actores privados, se revaloran las formas urbanas comercializables, redituables para el mercado (Janoschka, 2002, p. 11). El suelo urbanizable pierde importancia como recurso y, más que un bien de uso, se convierte en un capital, del cual el propietario puede obtener enormes beneficios.

El gusto por vivir en el campo ha generado la ocupación del suelo rural para usos urbanos: se han establecido fraccionamientos en los contextos de pequeńos asentamientos rurales, que propician una convivencia social entre los habitantes rurales (originarios) y los nuevos habitantes (campestres) exógenos a la comunidad.

La ocupación del territorio de las zonas periurbanas de las ciudades involucra aspectos sociales de las comunidades rurales, por la convivencia comunitaria con los pobladores de los asentamientos humanos que históricamente se mantuvieron separados de los polígonos de los centros de población urbana. La sobrevivencia de la organización ejidal ha desempeńado un papel fundamental en la permanencia de los vínculos familiares y comunitarios de población (Pepín, 1996, p. 69). Estas interacciones sociales muestran una dinámica de ocupación y convivencia entre los grupos de usuarios, o sea, entre habitantes originarios de la comunidad rural y los nuevos habitantes, con estatus socioeconómico, expectativas y formas de vida distintas.

Mediante estudios de las condicionantes físicas, geográficas y espaciales de las zonas periurbanas, se puede determinar el grado de impacto que se ha causado en el contexto rural en donde se ubican, por lo que se puede conocer el porcentaje de la extensión territorial destinada a actividades agropecuarias que han visto alterado su uso con fines de ocupación urbana. Esta situación, denominada "urbanización del campo", ha llevado a una intensa conversión del uso del suelo rural, así como el desplazamiento de las 
actividades primarias por las secundarias y terciarias (Ramírez, 1995, p. 17); por tal motivo, será de suma importancia conocer las situaciones específicas de los distintos escenarios de conurbación, en cuanto a su proximidad y la facilidad de interacción entre su suelo urbano y su suelo rural.

Otro de los motivos de la incursión de actividades urbanas en territorios rurales son los fraccionamientos de tipo campestre, los cuales no necesariamente satisfacen la demanda de los sectores sociales de menores ingresos; por lo contrario, el mercado objeto de los fraccionamientos en el campo ha sido la creación de desarrollos para la población de ingresos medios y altos, clubes hípicos y de golf, turismo y parques industriales (Olivera, 2004, p. 27).

En la caracterización de las nuevas operaciones residenciales en un sector de la periferia de la ciudad argentina de Rosario, Bragos y otros autores (2002) seńalan la aparición de los countries', asociada con el completamiento y la extensión de la planta urbana de Rosario y de aquellas localidades que siempre se han caracterizado por ser el lugar de la segunda residencia de sus pobladores. Se les llama "lugares de vivienda de fin de semana".

En América del Sur, en los emprendimientos del tipo countries los habitantes aspiran a tener un estilo de vida campestre, alejado del bullicio de la ciudad. Este tipo de emprendimiento se caracteriza por ser una organización territorial en torno a una o varias actividades deportivas; de allí que los diferentes espacios se articulen con los espacios destinados para tales actividades.

Estas áreas de uso comunitario adquieren mucha importancia. Los lagos propios, la red irregular de calles internas, la parcelación en grandes lotes ${ }^{6}$, las edificaciones grandes y lujosas y muchas otras campestres demuestran un estilo de vida orientado a disfrutar del tiempo libre (Ortiz y Cardoso, 2006, p. 10).

La conceptualización sobre la urbanización del campo ofrece un acercamiento al conjunto de fenómenos que se evidencian en el territorio rural. Abordar al territorio desde distintas perspectivas de estudio, a las zonas de reserva territorial, a la ocupación y al aprovechamiento del suelo por el hábitat humano en un contexto periurbano plantea una comprensión del traslado poblacional hacia el campo, como una alternativa ante la calidad de vida que hay en las ciudades y la ocupación del territorio de las zonas periurbanas.

\section{Enfoques de investigación}

Debido a la escasez de información teórica para abordar la manifestación del fenómeno de la urbanización del campo, este apartado se fundamenta en dos enfoques: el primero es el desarrollo endógeno. Desde los inicios de la década del ochenta aparece esta interpretación que surge de la confluencia de dos líneas de investigación: desarrollo de localidades rezagadas y desarrollo industrial al sur de Europa (Vázquez, 2007, p. 174). La primera nace como consecuencia del intento de encontrar una noción de desarrollo que permitiera actuar para lograr el desarrollo de localidades y territorios retrasados y la segunda como resultado del análisis de los procesos de desarrollo industrial endógeno en localidades y regiones del sur de Europa.

Endógeno significa "crecer desde adentro"; por lo tanto, el desarrollo endógeno trata de potenciar los recursos, las iniciativas y estrategias propias, así como la aplicación de conocimientos y sabidurías rurales en el proceso de desarrollo. Esta definición se fortalece con el esquema de la organización territorial de la producción, en el 
que se alistan las redes de empresas y las redes de actores en el análisis económico de los territorios y el reconocimiento de los componentes socioculturales e institucionales con valor estratégico en los procesos de desarrollo.

Los estudios sobre las mediciones del desarrollo endógeno y desarrollo sustentable tienen relación con estándares de medición específicos, caracterizados, según algunos autores, como indicadores e índices de sustentabilidad, que son mediciones del desempeño de los sistemas en el manejo de los recursos naturales. Müller (1996) los define como parámetros que proporcionan información sobre el estado actual de los ecosistemas y como patrones o tendencias en el estado del ambiente, en las actividades humanas que afectan o están afectadas por este o en las relaciones entre tales variables dentro de un ámbito territorial determinado.

La mayoría de los estudios revisados sobre el desarrollo en general integran la medida de la calidad de vida mediante indicadores ${ }^{7}$, utilizados en mediciones de la percepción de los habitantes en contextos urbanos. Estos indicadores requieren un análisis para su aplicación en el territorio rural.

El concepto de calidad de vida aparece como una alternativa por la que las personas deciden establecer viviendas campestres en contextos territoriales rurales. Su definición, según la Organización Mundial de la Salud (OMS), es la percepción que un individuo tiene de su lugar en la existencia, en el contexto de la cultura y del sistema de valores y en relación con sus expectativas, sus normas e inquietudes. Se trata de una noción muy amplia que está influida de modo complejo por la salud física del sujeto, su estado psicológico, su independencia, sus relaciones sociales y su relación con los elementos esenciales del entorno.
Las definiciones y conceptualizaciones encontradas en los estudios de calidad de vida incluyen elementos objetivos y subjetivos. Al concepto del bienestar material se integran la salud, el bienestar subjetivo, la seguridad, la calidad del ambiente, la educación, el buen gobierno, la democracia y otros. La calidad de vida es una alternativa para la medición del desarrollo rural, es decir, la consulta sobre la percepción de la gente, respecto a los índices del bienestar de los habitantes que se relacionan para convivir en un territorio común.

En el marco de la calidad de vida, dentro del abordaje territorial de la sustentabilidad, para Hernández (2009) se requiere la incorporación de este paradigma en un sistema de determinación y evaluación de los objetos y programas, en forma de un sistema de indicadores. En él, la calidad de vida está formada por tres dimensiones básicas: la calidad ambiental ${ }^{8}$, el bienestar ${ }^{9} \mathrm{y}$ la identidad ${ }^{10}$.

El bienestar como indicador de la calidad de vida es entendido como el estado por el cual una persona se "siente satisfecha con su vida". Algunas definiciones indican la "situación de ser feliz, saludable o próspero” (García, 2011, p. 75). Para algunos autores, debe separarse entre lo objetivo y lo subjetivo: mientras el primero incluye todos los elementos extrínsecos que coadyuvan a la satisfacción del individuo, el segundo se refiere a los elementos internos de la persona (García, 2011, p. 82). En todo caso, se deduce que el bienestar es un estado ideal al cual aspiran, en general, todos los seres humanos.

De acuerdo con Salvador Rueda (1996) las acotaciones a la calidad de vida como objeto de la representación social, para abordar la aproximación empírica del estudio de la representación social de la calidad de vida, es necesario limitar un ámbito particular dentro de todas las conceptualizaciones posibles de la calidad de vida. 
En México se han medido los índices de desarrollo de las regiones territoriales de los estados de la República, con base en indicadores de calidad de vida. El índice nacional de calidad de vida para México (Incavi) ${ }^{11}$ propone utilizar la calidad de vida como medida de desarrollo de los estados. Este índice es construido a partir de la percepción de los ciudadanos. Los dominios de calidad de vida considerados para la composición de los índices contemplan siete dominios de vida del ciudadano: salud ${ }^{12}$, economía ${ }^{13}$, educación ${ }^{14}$, se$\operatorname{guridad}^{15}$, buen gobierno ${ }^{16}$, vida comunitaria ${ }^{17} \mathrm{y}$ bienestar personal ${ }^{18}$ (García, 2011, p. 87).

El Incavi da las pautas para diagnosticar los índices de desarrollo de los estados del país. Mediante la valoración numérica de cada punto revisado se puede asignar una ponderación a la percepción de los ciudadanos con relación a los ejes de la calidad de vida que se proponen. Este instrumento requiere un ajuste para su utilización en el territorio rural; por medio de la adaptación y del replanteamiento de las preguntas específicas sobre la calidad de vida dirigidas a los habitantes de las comunidades rurales en las que se manifiesta el fenómeno de la urbanización del campo se podrá determinar, desde su percepción, el grado de los impactos generados en el desarrollo rural comunitario.

El segundo enfoque teórico de este trabajo consiste en las premisas en las modificaciones del ambiente territorial y social fundamentadas en los preceptos que propone la "ambitectura"19, los cuales permiten tener una visión sobre el espacio rural, a partir de una comprensión de los procesos de transformación en el territorio y en las sociedades de asentamientos humanos rurales.

La ambitectura muestra un sumario de experiencias prácticas, propuestas para su aplicación en el desarrollo urbano/ambiental de ciudades del continente americano desde una comprensión de temas sobre la forma urbana, que constituyen una teoría del ambiente construido. La ambitectura está más orientada a incluir cualquier transformación en el ambiente natural y social.

Los indicadores de la ambitectura proponen una metodología práctica basada en la conceptualización del territorio. Pesci (2007) sugiere un camino hacia un tratado de ambitectura y plantea los fundamentos para el desarrollo urbano sustentable sobre una propuesta teórica del movimiento arquitectónico/urbanista en América Latina.

A partir de esta visión de estructura territorial tras un análisis macrorregional del espacio, es posible determinar el funcionamiento sistémico de las comunidades emplazadas en zonas rurales, así como las interacciones que se establecen con el asentamiento humano mayor, es decir, la caracterización espacial de las comunidades rurales en su proximidad a la zona urbana. De lo anterior se vislumbra la importancia de revisar los focos sociales y económicos presentes en el territorio, debido a que la convivencia socioterritorial genera tensiones en el desarrollo local que pueden analizarse desde la información social, cultural, económica y educativa de la región.

8 Los indicadores de esta área remiten a calidad ambiental de los espacios, consumos de recursos naturales, suelo y energía y emisión y tratamiento de los residuos.

9 Es una construcción social, cuyo grado de satisfacción se mide por comparación con un nivel de referencia, asociado con parámetros administrativos que para ser comparables, deben ser homogenizados.

10 Trata de evaluar la participación social en el territorio, la utilización del tiempo y el espacio por parte de los habitantes y la satisfacción de sus necesidades subjetivas.

11 Este índice fue elaborado en 2008 por el Centro de estudios sobre el bienestar de la Universidad de Monterrey, junto con investigadores del Centro de estudios sociales y de opinión pública (Cesop) de la Cámara de Diputados de México.

12 Estado de salud. Veces que ha visitado al doctor en los últimos seis meses. Calidad de los servicios médicos.

13 Si el dinero cubre necesidades básicas de alimentación. Facilidad para conseguir casa de habitación apropiada. Viabilidad de conseguir trabajo adecuado.

14 Calidad académica de las escuelas. Acceso a buena educación y eventos culturales, deportivos y de esparcimiento.

15 Seguridad en la comunidad. Si ha sido víctima de la inseguridad en los últimos doce meses. Capacidad de las autoridades para enfrentar el fenómeno.

16 Honestidad y eficiencia de los gobiernos (municipal, estatal, federal). Calidad de los servicios públicos.

17 Clima. Calidad del ambiente. Calidad de los servicios no gubernamentales. Facilidad para moverse alrededor de la ciudad.

18 Disponibilidad de tiempo libre. Percepción de su calidad de vida. Deseo de pasar el resto de la vida en la comunidad. Facilidad para convivir con familiares y amigos.

19 Como propuesta teórica de conceptualización del territorio para el análisis de transformaciones en el ambiente territorial y social. 
Esta visión de análisis basada en una estructura antrópica se refiere a la investigación del habitante, o sea, fundada en una escala humana; por lo tanto, al considerar la percepción de los habitantes de territorios donde se manifiesta el fenómeno de la urbanización del campo, se tendrá información de utilidad para la fundamentación de las distintas visiones de los habitantes en su interacción social y territorial.

De la revisión teórica conceptual se define el desarrollo comunitario del territorio rural como la liga conceptual principal, en la que se integran los dos enfoques de la investigación. El primero respecto al desarrollo comunitario rural, luego del análisis de los procesos de desarrollo en las comunidades en el que se involucran actores urbanos y rurales dentro de la organización territorial (Tabla 1).

Tabla 1.

Variables de investigación desde el enfoque

del desarrollo comunitario rural

\begin{tabular}{|c|c|c|}
\hline \multirow{6}{*}{ 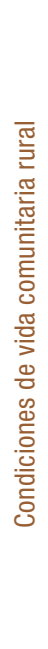 } & \multicolumn{2}{|c|}{$\begin{array}{l}\text { Interacciones comunitarias de agrupación } \\
\text { (ecoforma en microescala) }\end{array}$} \\
\hline & Indicador & Instrumento \\
\hline & $\begin{array}{l}\text { Forma de agrupación } \\
\text { de la comunidad } \\
\text { (dispersa / agrupada). }\end{array}$ & $\begin{array}{l}\text { Observación directa / } \\
\text { Visita de campo Ficha }\end{array}$ \\
\hline & $\begin{array}{r}\text { Condiciones de } \\
\text { (socioforme }\end{array}$ & $\begin{array}{l}\text { de los habitantes } \\
\text { nicroescala) }\end{array}$ \\
\hline & Indicador & Instrumento \\
\hline & $\begin{array}{l}\text { Salud, Economía, Educa- } \\
\text { ción, Seguridad, Autoridad } \\
\text { y gobierno, Vida comunita- } \\
\text { ria, Accesibilidad y movili- } \\
\text { dad, Bienestar personal. }\end{array}$ & Cuestionarios \\
\hline
\end{tabular}

Fuente: elaboración propia

El segundo enfoque, mediante la revisión de las transformaciones territoriales, espaciales y sociales sobre los preceptos teóricos que dan las pautas para revisar, desde un entendimiento sistémico, la organización territorial con interacción campo-ciudad (Tabla 2).
Tabla 2.

Variables de investigación desde el enfoque

de las transformaciones en el ambiente territorial y social

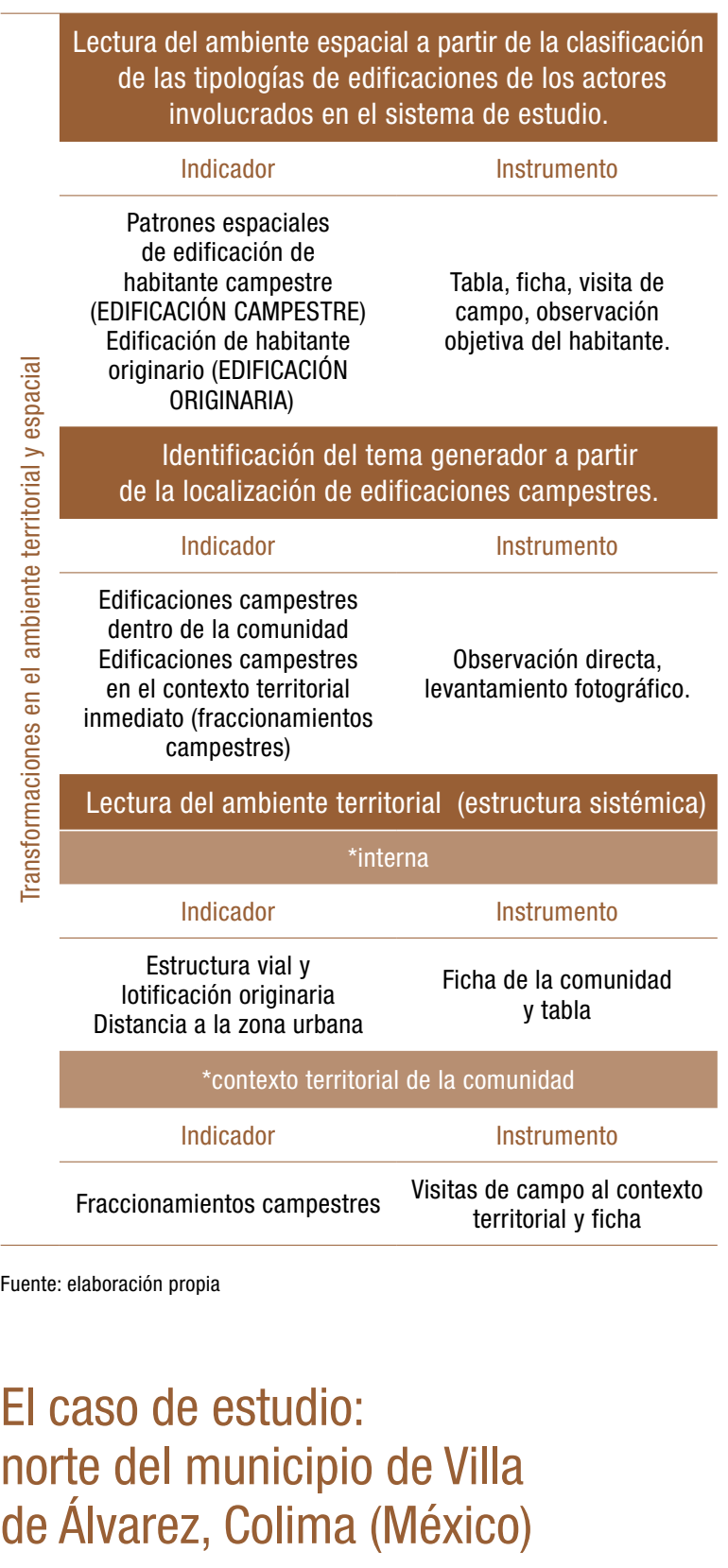

En la conurbación Colima-Villa de Álvarez se identifica una expansión fragmentada no conurbada, debido a que en el contexto territorial norte existen comunidades rurales tradicionalmente asentadas en una zona apta para la actividad agrícola en donde se han detectado procesos urbanos de ocupación del territorio con diversos usos de suelo. 
El estado de Colima se localiza al occidente de la República mexicana. Es el cuarto estado más pequeño en cuanto a sus dimensiones territoriales. Colinda al norte con el estado de Jalisco, al sur con el estado de Michoacán y al oeste con el océano Pacífico. La ciudad capital del estado recibe el mismo nombre: Colima. Políticamente se divide en diez municipios: Armería, Colima, Comala, Coquimatlán, Cuauhtémoc, Ixtlahuacán, Manzanillo, Minatitlán, Tecomán y Villa de Álvarez.

La principal vía de comunicación hacia el interior del país se origina en el puerto localizado en el municipio costero de Manzanillo y atraviesa el territorio estatal.

También existe una red de interconexión carretera de escala local que comunica a los poblados rurales que han permanecido inmersos en contextos territoriales y paisajísticos rurales (Figura 1).
El municipio de Villa de Álvarez se encuentra ubicado entre las coordenadas extremas de los paralelos $19^{\circ} 15^{\prime}$ a $19^{\circ} 21^{\prime}$ de latitud norte y $103^{\circ}$ $40^{\prime}$ a $104^{\circ} 05^{\prime}$ de longitud oeste del meridiano de Greenwich, a una altitud máxima de $1.600 \mathrm{msnm}$ y una mínima de 440. Limita al norte con el municipio de Comala; al suroeste, con el de Colima; al noroeste, con el de Minatitlán; al suroeste, con el de Coquimatlán y al este con el de Cuauhtémoc. En la delimitación de las zonas metropolitanas de México del Consejo Nacional de Población (Conapo) se integra a la zona metropolitana de Colima-Villa de Álvarez la población de los municipios de Colima, Comala, Coquimatlán, Cuauhtémoc y Villa de Álvarez (Conapo, 2012).

El asentamiento poblacional de mayor concentración demográfica corresponde al territorio conurbado de la capital del estado y la zona urbana del municipio de Villa de Álvarez. La población total en ambos municipios, hasta 2010, es de 266.860 habitantes (Conapo, 2012) (Tabla 3).

Figura 1.

Red de infraestructura de carreteras y contexto territorial del estado de Colima

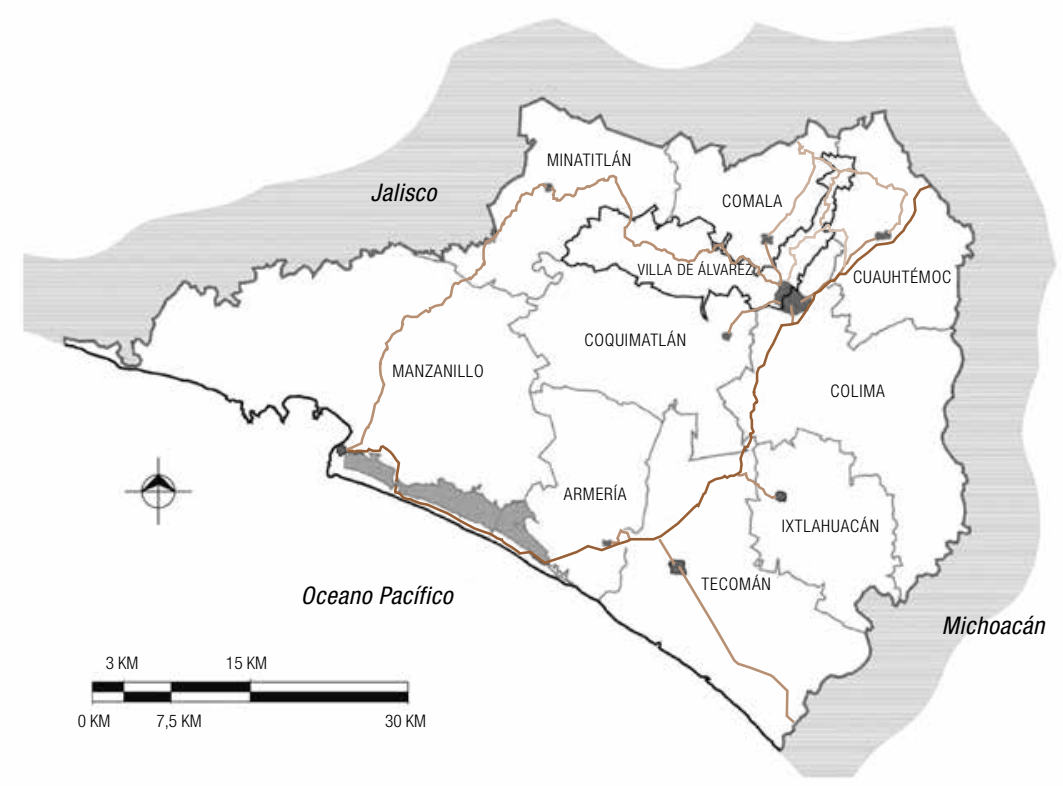

Fuente: elaboración propia con base en el plano del estado Colima de la Secretaría de Salud del Gobierno del estado de Colima (2012) 
Tabla 3.

Zona metropolitana de Colima-Villa de Álvarez: población, tasa de crecimiento, superficie y densidad media urbana, 1990-2010

\begin{tabular}{|c|c|c|c|c|c|c|c|c|}
\hline \multirow[t]{2}{*}{ Clave } & \multirow[t]{2}{*}{ Municipio } & \multicolumn{3}{|c|}{ Población } & \multicolumn{2}{|c|}{$\begin{array}{c}\text { Tasa de crecimiento } \\
\text { medio anual }(\%)\end{array}$} & \multirow{2}{*}{$\begin{array}{l}\text { Superficie }{ }^{1} \\
\qquad\left(\mathrm{~km}^{2}\right)\end{array}$} & \multirow{2}{*}{$\begin{array}{l}\text { DMU² }^{2} \\
\text { (hab/ha) }\end{array}$} \\
\hline & & 1990 & 2000 & 2010 & $1990-2000$ & $2000-2010$ & & \\
\hline \multicolumn{2}{|c|}{$\begin{array}{c}\text { Zona metropolitana } \\
\text { de Colima-Villa de Álvarez }\end{array}$} & 211733 & 275677 & 334240 & 2,7 & 1,9 & 2287,6 & 68,2 \\
\hline 6002 & Colima & 116505 & 129958 & 146904 & 1,1 & 1,2 & 746,0 & 62,7 \\
\hline 6003 & Comala & 16909 & 19384 & 20888 & 1,4 & 0,7 & 314,3 & 38,9 \\
\hline 6004 & Coquimatlán & 16019 & 18756 & 19385 & 1,6 & 0,3 & 527,0 & 50,1 \\
\hline 6005 & Cuauhtémoc & 24458 & 26771 & 27107 & 0,9 & 0,1 & 412,3 & 39,4 \\
\hline 6010 & Villa de Álvarez & 37842 & 80808 & 119956 & 7,9 & 3,9 & 288,0 & 85,3 \\
\hline
\end{tabular}

${ }^{1}$ El dato de Superficie se obtuvo de las Áreas Geoestadísticas Municipales (AGEM), del Marco Geoestadístico Nacional 2010.

${ }^{2}$ Densidad Media Urbana: El dato de superficie para el cálculo de la DMU se obtuvo a partir de las Áreas Geoestadísticas Básicas (AGEB) urbanas, de la Cartografía Geoestadística Urbana del Censo de Población y Vivienda 2010.

Nota: Los límites estatales y municipales fueron compilados del marco geoestadístico del INEGI, el cual consiste en la delimitación del territorio nacional en unidades de áreas codificadas, denominadas Áreas Geoestadísticas Estatales (AGEE) y Áreas Geoestadísticas Municipales (AGEM), con el objeto de referenciar la información estadística de censos y encuestas. Los límites se apegan en la medida de lo posible a los límites político-administrativos.

Fuente: Conapo (2012)

El entorno rural norte de la zona urbana tiene una localización geográfica en ladera; esto ha generado que el sistema de vialidades regionales permita una accesibilidad inmediata a una zona más elevada en cuanto a la altura sobre el nivel medio del mar que tiene el valle. Existe una red de interconexión vial de escala local con una configuración concéntrica a la principal zona urbana, que comunica a los poblados rurales de la zona.

En la parte norte, el territorio municipal de Villa de Álvarez comprende una franja espacial con delimitación entre los municipios de Comala al poniente y Cuauhtémoc al oriente. La zona rural del norte del municipio de Villa de Álvarez (NMVA) es una cuenca territorial con facilidad de acceso por la carretera Villa de Álvarez-El Naranjal (Figura 2).

El caso de estudio se define a partir de la localización de seis comunidades rurales que se ubican dentro de la escala geográfica generada por la expansión fragmentada no conurbada a la ciudad Colima-Villa de Álvarez, en donde se ha detectado la presencia de viviendas campestres entre las comunidades originarias y en fraccionamientos campestres en sus contextos inmediatos (Figura 3).

A partir de las lecturas del ambiente territorial y espacial de las comunidades en la zona rural NMVA, se puede concluir que del total de comunidades analizadas en su funcionamiento como sistema de comunidades rurales, estas poblaciones se consideran asentamientos con población menor a 2.500 habitantes. Según el censo de población y vivienda del año 2010, la población total en las seis unidades de estudio corresponde a comunidades rurales con población total menor a doscientas personas. De igual forma, cumple con la característica de presentar una densidad poblacional menor a cinco habitantes por hectárea.

En cuanto a la ocupación espacial de los predios o terrenos y las edificaciones construidas en las zonas habitacional campestre, tipo h1, en el Artículo 54 del Reglamento de zonificación del estado de Colima se especifican los lineamientos para la configuración de los terrenos campestres, con una densidad máxima de treinta habitantes por hectárea. 
Figura 2.

Contexto de ubicación de las comunidades rurales del norte del municipio de Villa de Álvarez

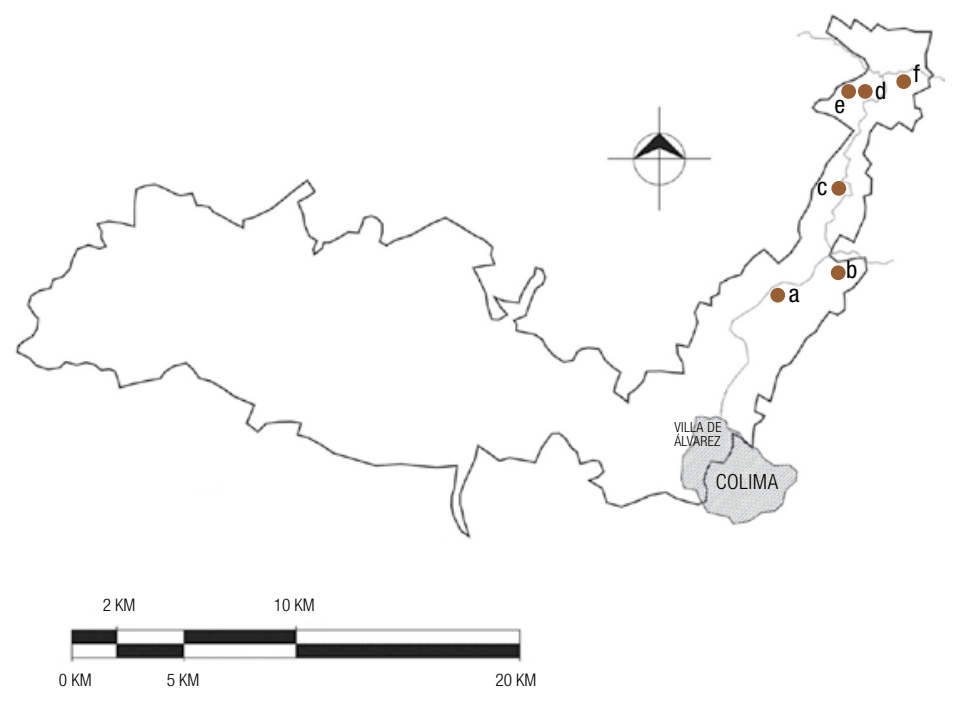

Fuente: elaboración propia con base en el plano del estado Colima de la Secretaría de Salud del Gobierno del estado de Colima (2012)
Figura 3.

a) El Chivato, b) Las Joyitas, c) El Carrizal,

d) Nuevo Naranjal, e) La Lima, f) El Naranjal

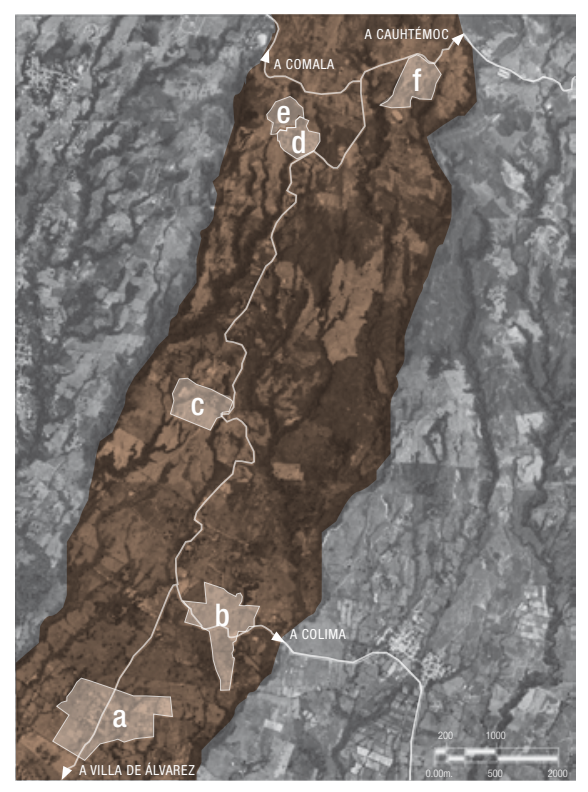

Fuente: elaboración propia sobre imagen satelital del Instituto Nacional de Estadística y Geografía (INEGI) (2012)
Con base en esta consideración, se puede confirmar que la misma legislación urbana permite densificar el territorio de manera tal que los fraccionamientos campestres puedan ser territorialmente mayores —en cuanto a sus dimensiones y densidad poblacional- que las comunidades rurales que históricamente han permanecido en el territorio. Esta situación hace inminente la necesidad de generar los instrumentos legales aplicables para contextos rurales y no urbanos concebidos desde la perspectiva de aplicación.

En el esquema territorial de cada localidad se puede apreciar la estructura interna y contextual, así como la superficie en hectáreas de cada asentamiento. La forma de agrupación en el territorio es concentrada en el espacio del asentamiento originario. Existe una red vial básica de configuración que define la lotificación de la comunidad y en algunas, en el contexto territorial inmediato, se aprecia la edificación de residencias de tipo campestre en una forma dispersa, es decir, con grandes dimensiones territoriales, en ocasiones mayores al asentamiento originario.

De la lectura del ambiente espacial se puede afirmar que los asentamientos originarios se emplazan a lo largo de la carretera que comunica con el centro urbano de Villa de Álvarez. Se identifican algunos equipamientos como referentes de congregación social de las comunidades; los jardines públicos y los templos son los espacios comunes de interacción socioespacial. Con origen en el núcleo central de la comunidad, se dispersa espacialmente el emplazamiento con la disposición territorial para fraccionamientos de tipo campestre en los contextos inmediatos.

Respecto a la morfogénesis del territorio, se identificó que en los polígonos de las comunidades analizadas hay un trazado urbano inicial en donde se ubican las edificaciones propias. En lo que respecta al crecimiento espacial del origen del asentamiento, se aprecia el respeto por 
las condiciones de topografía, masa vegetal y presencia de escurrimientos naturales.

La evaluación de los indicadores espaciales de permeabilidad y legibilidad es muy baja, debido a que las comunidades han seguido un proceso de desarrollo y expansión sin planificación territorial y disponen de los recursos inmediatos con los que se cuenta; esta situación repercute en detrimento de las relaciones sociales comunitarias y, por lo tanto, en el desarrollo endógeno de la región.

De los patrones espaciales de las edificaciones originarias en cuanto al tamaño de los predios y las edificaciones, se constató el cumplimiento de los coeficientes de ocupación y uso para zonas unifamiliar de densidad baja tipo $\mathrm{H} 2$, debido a que la morfología de edificaciones es de una planta, los materiales más utilizados son tabique rojo y tabicón en muros, losa plana de concreto armado y cubiertas inclinadas con lámina galvanizada.

Los predios originarios no tienen banquetas en la vía pública y, en el espacio destinado para tal, se dispone de vegetación de ornato y arbolado. De igual forma, se infringe con el lineamiento de dos cajones para estacionamiento dentro del predio, debido, sobre todo, a que los habitantes oriundos utilizan el transporte público para traslados a la zona urbana.

De los patrones de edificaciones campestres se identificó la característica de altura de edificaciones de dos plantas como la diferencia más significativa respecto a las edificaciones originarias de una planta. En su mayoría, se cumple con los lineamientos del Reglamento de zonificación del estado de Colima para zonas habitacionales campestres tipo $\mathrm{H} 1$, salvo algunas excepciones respecto a cuatro cajones para estacionamiento dentro del predio, restricciones de edificación y modo de edificación, puesto que había predios con edificaciones campestres amurallados en sus límites, situación que genera una fisonomía espacial opuesta a la originaria que se mimetiza con el verde de la vegetación.

Del comportamiento de la población se puede afirmar que las comunidades han mantenido un crecimiento natural de su población. En las diferencias porcentuales de crecimiento de los últimos dos censos, se detalla un incremento de la población en El Chivato, Las Joyitas y La Lima y una relativa disminución en El Carrizal, Nuevo Naranjal y El Naranjal. Con estos datos porcentuales del crecimiento poblacional, se puede establecer que, en últimas fechas, la inserción de habitantes campestres al territorio de las comunidades ha aumentado en las localidades más cercanas a la conurbación Colima-Villa de Álvarez y en donde se detectan fraccionamientos campestres en su contexto territorial inmediato.

Como punto principal para la determinación de la muestra estadística y para el cumplimiento del objetivo particular de investigación - que consiste en evaluar y contrastar los satisfactores de la percepción de las condiciones de vida del habitante originario y del habitante campestre- se consideraron tres comunidades que presentan al menos un fraccionamiento campestre: El Chivato, Las Joyitas y El Carrizal, como comunidades rurales con fraccionamientos campestres en contexto territorial (predominancia campestre) y las otras tres comunidades, Nuevo Naranjal, La Lima y El Naranjal, como comunidades rurales con edificaciones campestres dentro del asentamiento (predominancia originaria).

\section{Discusión de datos}

En cuanto a la valoración que los mismos habitantes hicieron sobre ocho $^{20}$ indicadores de las 
condiciones de vida en la región, se otorgó una valoración numérica ${ }^{21}$ a su evaluación. Los habitantes originarios presentaron una ponderación mayor en los términos de vida comunitaria y bienestar personal y la ponderación menor en el rubro educativo (Figura 4).

Figura 4.

Percepción de los habitantes originarios sobre los indicadores de calidad de vida en comunidades con predominancia campestre
Originarios
Fuente: elaboración propia con base en cuestionarios aplicados entre junio de 2012 y enero de 2013

Los habitantes campestres evaluaron con mayor puntaje el bienestar personal y la accesibilidad y movilidad y puntajes menores en los rubros de educación y seguridad (Figura 5).

Figura 5.

Percepción de los habitantes campestres sobre los indicadores de calidad de vida en las comunidades con predominancia campestre

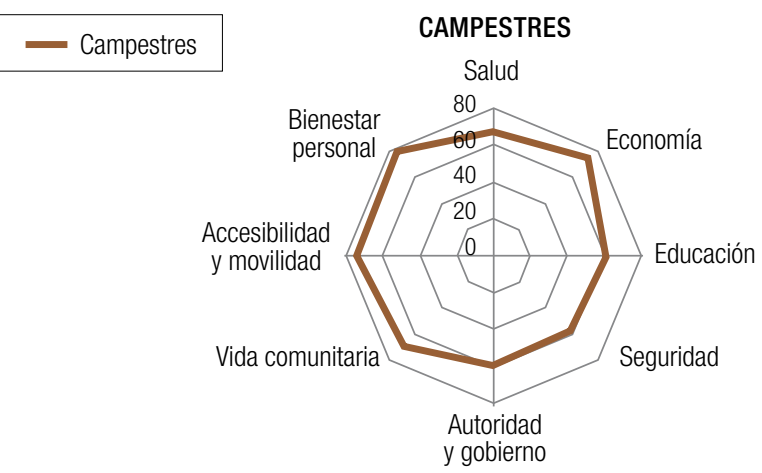

Fuente: elaboración propia con base en cuestionarios aplicados entre junio de 2012 y enero de 2013

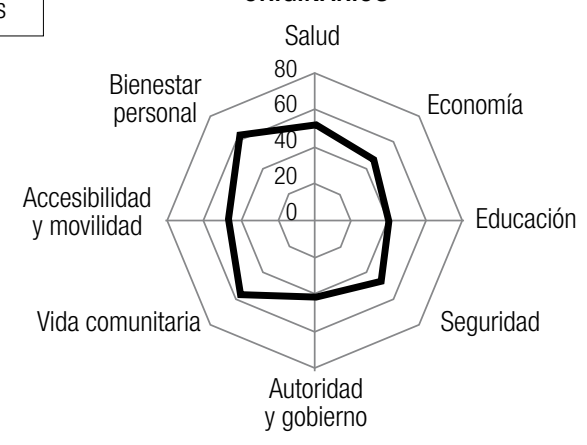

Después de conocer los resultados cuantitativos de las ponderaciones, mediante una correlación porcentual de la información se definieron gráficamente los polígonos cerrados de frecuencias relativas acumuladas para cada tipo de habitante y se compararon los porcentajes con mayor y menor valoración en cada caso (Figura 6).

Figura 6.

Percepción de los habitantes originarios y los habitantes campestres sobre los indicadores de calidad de vida en las comunidades con predominancia campestre

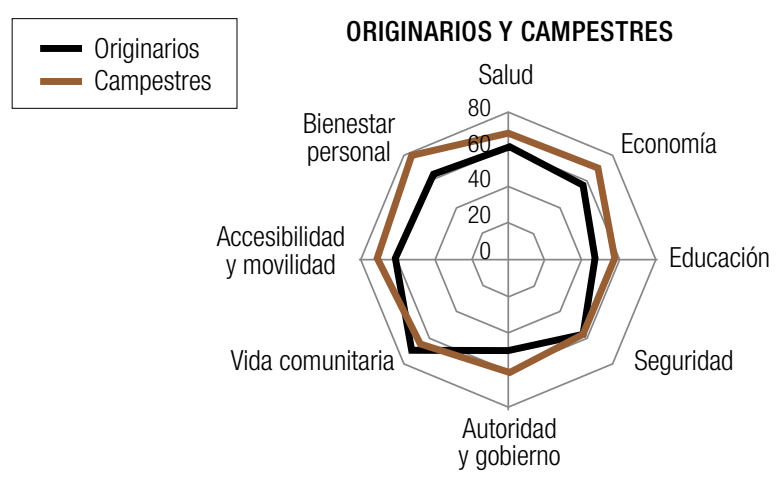

Fuente: elaboración propia con base en cuestionarios aplicados entre junio de 2012 y enero de 2013

Como resultado de esta correlación de datos, se pueden interpretar, a partir de la participación social de los actores involucrados en los conflictos y potencial del ambiente de estudio, las transformaciones socioterritoriales surgidas por las relaciones establecidas entre dichos actores.

En la definición de la morfogénesis desde la lectura de la socioforma de ambos habitantes, se señala que perciben de distinto modo los satisfactores de las condiciones de vida; los rubros de seguridad, salud y vida comunitaria presentan los valores más aproximados y en los índices de economía, educación, accesibilidad y movilidad y autoridad y gobierno muestran las disparidades más significativas.

La percepción de bienestar personal es la que reveló la diferencia más significativa entre ambos actores, debido, sobre todo, a los señalamientos 
de los habitantes originarios respecto a su deseo por radicar en la ciudad y en sentido opuesto el deseo de los campestres por radicar en el campo.

\section{Conclusiones y recomendaciones}

Tras el análisis y la discusión de los datos, se puede afirmar que, como se propuso en la hipótesis, existe una dicotomía acerca de la percepción de ambos tipos de habitantes, situación cierta hasta un punto, puesto que ambos grupos tienen una apreciación distinta del espacio-territorio: mientras los originarios no han tenido acceso al bienestar urbano, los campestres están acostumbrados a los servicios urbanos que les resultan deficientes en el contexto rural.

Luego de las lecturas del ambiente territorial de la ecoforma y de la socioforma en la zona, se evidenció que la disposición temporal y permanente del suelo para usos campestres afecta negativamente los procesos rurales de la región al ser constituidos como polígonos aislados en el territorio (con un solo acceso y egreso desde la carretera o que atraviesan terrenos agrícolas), por el limitado control municipal en cuanto al seguimiento para la regulación legal de los mismos y por las obras mínimas de urbanización requeridas para su origen.

En lo espacial, por la identificación de relaciones no convenientes, por la baja permeabilidad y legibilidad de las comunidades y por la expansión territorial que generan los fraccionamientos campestres en los contextos territoriales de los asentamientos originarios. Sin embargo, como indicadores positivos en la lectura de los patrones espaciales, se detectó el respeto por el medio circundante, puesto que los trazos viales respetan la topografía natural del terreno, cauces de escurrimientos, arroyos y macizos arbolados.

En lo social, se demostró que los habitantes campestres se mantienen ajenos al proceso de desarrollo comunitario, pues sus necesidades están vinculadas a funciones urbanas, mientras los originarios conservan costumbres propias del campo y se mantienen ajenos e indiferentes ante la presencia de nuevos habitantes, a quienes no consideran miembros de la comunidad; por lo contrario, expresaron llevar una mala relación por afectaciones en la convivencia.

Sobre las diferencias encontradas en las percepciones de los habitantes ligadas con las condiciones de vida en la región, se expresó gráficamente que, en las comunidades con predominancia campestre, se manifiestan con mayor claridad las disparidades entre originarios y campestres, sobre todo por los habitantes campestres con residencia permanente en el sitio y que están unidos al ámbito urbano laboral; esto hace que el vínculo entre el territorio urbano y rural sea mínimo, al disponer de vehículos automotores que han reducido el tiempo de traslado entre el campo y la ciudad.

En las comunidades con predominancia originaria, las valoraciones sobre las condiciones de vida coincidieron casi en todos los rubros, con excepción del aspecto económico, indicador que sirvió para evidenciar la dicotomía en cuanto al poder adquisitivo de los adultos mayores, quienes conforman la mayoría de los habitantes de las comunidades más alejadas a la zona urbana.

En cuanto a la caracterización del fenómeno socioterritorial, se comprobó el supuesto, debido a que los procesos de desarrollo rural de las comunidades están siendo afectados por el fenómeno de la urbanización del campo.

En la revisión teórica referente al desarrollo endógeno comunitario se señaló que este trata de potenciar recursos, iniciativas y estrategias propias, así como la aplicación de conocimientos y sabidurías rurales en el proceso de desarrollo. Para el caso de estudio, se hizo evidente que no existe tal desarrollo en las comunidades rurales, porque las condiciones de convivencia e interacción son muy limitadas, situación aprovechada 
por habitantes campestres para beneficio particular y no común.

Al comparar entre las comunidades con predominancia campestre con las de predominancia originaria, como principal factor de diferencia puede determinarse la accesibilidad por carretera, ya que la red de vialidades rurales ha permitido a personas exógenas al medio rural que dispongan de este territorio por la reducción temporal que implica el traslado campo-ciudad y viceversa; esto ha hecho de las comunidades con mayor presencia de residencias campestres de uso permanente las de menor distancia a la zona urbana, mientras las comunidades con predominancia originaria son alejadas de la ciudad.

Si bien se elaboró en este trabajo una caracterización de la disposición del suelo para usos campestres y un análisis del funcionamiento del sistema territorial de la zona y de la percepción de los habitantes del territorio rural del NMVA sobre las relaciones sociales y satisfactores en cuanto a las condiciones de vida en la región, hace falta complementar el eje económico y ambiental de la sustentabilidad:

a) En lo económico, es necesario hacer un estudio de mercado respecto a la oferta de predios campestres en la zona, así como una revisión de los promotores de desarrollos campestres, en cuanto al negocio que implica el mercado del suelo para fines de este uso. Esta situación genera otras tensiones respecto al compromiso legal que implica el proceso de municipalización de dichos fraccionamientos, el suministro y la dotación de servicios y el pago de predial y otros impuestos de carácter urbano en un medio inminentemente rural. b) En razón al eje ambiental, acerca del suministro de las instalaciones urbanas para el desarrollo de fraccionamientos campestres, ya que la apertura de vialidades para el tendido de redes involucra el derribamiento de macizos arbolados y la modificación de topografías accidentadas, barrancos y movimientos de tierras que alteran el paisaje natural del campo, la contaminación por ruido y, en general, la alteración de la biota endémica de la región.

Tras la realización de esta investigación se constató que el conjunto de comunidades de estudio, dentro de la estructura sistémica de la región, tiene una dinámica propia, es decir, asuntos, desafíos, necesidades, debilidades y también fortalezas, valores y oportunidades.

Es inminente la necesidad de elaborar una propuesta estratégica territorial para la urbanización del campo de la zona norte del municipio de Villa de Álvarez, la cual tendrá que considerar como objetivo primordial la identificación de estos aspectos y convertirse en un instrumento de referencia para la planeación comunitaria y territorial de los asentamientos humanos rurales de la región.

De los resultados de este trabajo se puede señalar una serie de recomendaciones: sobre la planeación territorial, que busque pasar de la visión administrativa municipal, que vigila y regula los procesos urbanos, a una visión territorial, que considere la correspondencia y el complemento entre el campo y la ciudad. Actuar bajo una visión territorial permitirá potenciar el desarrollo rural sustentable de la zona norte del municipio de Villa de Álvarez.

\section{Bibliografía}

Bazant, J. (2010). Expansión urbana incontrolada y paradigmas de la planeación urbana. Espacio Abierto, 19(3), 475-503.

Bragos, Ó., Mateos, A. y Pontoni, S. (2002). Nuevos desarrollos residenciales y procesos de segregación socio-espacial en la expansión oeste de Rosario. En L. Cabrales (coord.), Latinoamérica: paises abiertos, ciudades cerradas (pp. 441480). México D. F.: Universidad de Guadalajara, Unesco. 
Conapo. (2012). Delimitación de las zonas metropolitanas 2010. Recuperado de http://www. conapo.gob.mx/es/CONAPO/Zonas_metropolitanas_2010

Cruz, M. (1996). La urbanización ejidal. El encuentro de dos procesos rural y el urbano. En A. de Teresa y C. Cortés (coords.), La nueva relación campo-ciudad y la pobreza rural (pp. 123144). México D. F.: INAH AJAM-A, UNAM, P y V.

Cruz, M. (2002). Procesos urbanos y ruralidad en la periferia de la ZMCM. Estudios demográfcos y urbanos (49), 39-76.

Delgado, J. (2003). Transición rural-urbana y oposición campo-ciudad. En A. Aguilar (coord.), Urbanización, cambio tecnológico y costo social. El caso de la región centro de México. (pp. 77-118). México D. F.: IG-UNAM, Miguel Ángel Porrúa Editores.

Drescher, A. e Iaquinta, D. (2000) Defining Periurban: Understanding Rural-Urban Linkages and their Conection to Institutional Contexts. Manuscrito no publicado.

García, J. J. (2011). Hacia un nuevo sistema de indicadores de bienestar. Realidad, datos y espacio: Revista internacional de estadística y geografia, 2(2), 78-95.

Hernández, A. (2009). Calidad de vida y medio ambiente urbano. Indicadores locales de sostenibilidad y calidad de vida urbana. INVI, 24(65), 79-111.

Hiernaux, D. (2001). Las nuevas formas urbanas y reestructuración del mundo rural. En P. Torres (comp.), Procesos metropolitanos y agricultura urbana (pp. 19-30). México D. F.: UAM-X, FAO.
Janoschka, M. (2002). El nuevo modelo de la ciudad latinoamericana: fragmentación y privatización. EURE, 28(85). Recuperado de http:// www.redalyc.org/articulo.oa?id=19608502

Müller, S. (1996). ¿Cómo medir la sostenibilidad? Una propuesta para el área de la agricultura y de los recursos naturales. San José: GTZ, IICA.

Olivera, G. (2004). Trayectoria de las reservas territoriales en México: irregularidad, desarrollo urbano y administración municipal tras la reforma constitucional de 1992. EURE, 27(81). Recuperado de http://www.redalyc.org/articulo. oa?id=19608104

Ortiz de D’Arterio, J. L. y Cardozo Magalhaes, A. G. (2006). Periurbanización, segregación social y fragmentación territorial en San Miguel de Tucumán. Revista del Departamento de Geografia (11), 66-88.

Pepin, M. (1996). Entre ruralidad y urbanidad, la fuerza del lugar, En A. de Teresa y C. Cortés (coords.), La nueva relación campo-ciudad y la pobreza rural (pp. 69-82). México D. F.: INAH AJAM-A, UNAM, P y V.

Pesci, R. (2007). Ambitectura: Hacia un tratado de arquitectura, ciudady ambiente. La Plata: Ediciones $\mathrm{Al}$ margen.

Ramírez, B. (1995). La región en su diferencia: los valles centrales de Querétaro 1940-1990. México D. F: Red Nacional de Investigación Urbana.

Rueda, S. (1996). Habitabilidad y calidad de vida. Recuperado de http://polired.upm.es/index.php/ciur/article/view/1041/1060

Vázquez, A. (2007). Desarrollo endógeno. Teorías y políticas de desarrollo territorial. Investigaciones Regionales (11), 183-210. 